\title{
Influencia de la atmósfera de control y tiempo de molienda sobre la morfología y microestructura de polvos de cobre puro y cobre- $2,5 \%$ litio producidos por aleado mecánico ${ }^{(*)}$
}

\author{
P.A. Rojas*, **, M.P. Álvarez***, A. Peñaloza***, A. Zúñiga**** y S. Ordoñez****
}

\begin{abstract}
Resumen En el presente trabajo se investigó el efecto de la atmósfera y el tiempo de molienda sobre las fases, microestructura y composición de polvos de cobre y de una mezcla cobre $-2,5 \%$ litio, sometidas a aleado mecánico. La molienda se realizó en un molino SPEX 8000D empleando contenedores y bolas de acero. Las dos atmósferas estudiadas fueron argón y nitrógeno, y los tiempos de molienda de 3 a $30 \mathrm{~h}$. Los cambios microestructurales y las fases se analizaron mediante microscopía electrónica de barrido y difracción de rayos X, mientras que la composición química se determinó mediante espectroscopía de absorción atómica e infrarroja. Los resultados mostraron el efecto de los parámetros de molienda estudiados en la microestructura y en la composición química de las muestras.
\end{abstract}

\section{Influence of the control atmosphere and milling time on the morphology and microstructure of pure copper and copper-2.5 \% lithium powders produced by mechanical alloying}

\begin{abstract}
In the present work was investigated the effect of two milling parameters, atmosphere and milling time, on the morphology and microstructure of pure copper powder and a mixture of copper-2,5 wt. \% lithium. The mechanical alloying was performed in a SPEX 8000D mill, using steel containers and balls. The two control atmospheres were argon and nitrogen and the milling time was varied from 3 up to 30 hours. The microstructural changes and the phases after milling were analyzed using scanning microscopy and $\mathrm{X}$ ray diffraction, whereas the amount of iron was measured by atomic absorption spectroscopy and the amount of oxygen by infrared spectroscopy. The results show the effect of the milling parameters studied on the microstructure as well as on the chemical composition of the samples.
\end{abstract}

Keywords

Mechanical alloying; Copper-lithium alloys; Supersaturated alloys; powder metallurgy, X-ray diffraction.

\section{INTRODUCCIÓN}

El cobre y sus aleaciones poseen numerosas aplicaciones, destacando como materiales de uso en ingeniería en la industria eléctrica, sector que capta más del $75 \%$ del cobre producido a nivel mundial. Por otra parte, el litio ha cobrado importancia como material en ingeniería, pero no como base de aleaciones sino como elemento de aleación, principalmente en aleaciones base aluminio ${ }^{[1]}$. Chile posee yacimientos, importantes a nivel mundial, de ambos elementos que están ubicados en el sector norte del país, sin embargo, además de esta proximidad geológica, cobre y litio no poseen características físicas o químicas en común. Así, por ejemplo, mientras el cobre posee una densidad de $8,92 \mathrm{~g} / \mathrm{cm}^{3}$ y temperatura de fusión de $1.357 \mathrm{~K}$, el litio posee una densidad de $0,535 \mathrm{~g} / \mathrm{cm}^{3}$, su fusión se realiza a $453,69 \mathrm{~K}$ y su ebullición a $1.620 \mathrm{~K}$. Estas diferencias se manifiestan en, prácticamente, todas sus demás propiedades, aspecto que representa una complicación importante al momento de diseñar un proceso de elaboración para aleaciones $\mathrm{Cu}-\mathrm{Li}$, principalmente vía fusión. Sin

\footnotetext{
(•) Trabajo recibido el día 21 de noviembre de 2007 y aceptado en su forma final el día 14 de enero de 2009.

* Instituto de Física, Pontificia Universidad Católica de Valparaíso, Av. Brasil 2950, Valparaíso, Chile.

** Escuela de Ingeniería Mecánica, Pontificia Universidad Católica de Valparaíso, Av. Brasil 2950, Valparaíso, Chile.

*** Departamento de Ingeniería Mecánica, Universidad de Chile, Beaucheff 861, Santiago, Chile.

**** Departamento de Ingeniería Metalúrgica, Universidad de Santiago de Chile, Av. Libertador Bernardo O’Higgins 3363 , Santiago Chile.
} 
embargo, si se desarrolla un cálculo considerando las densidades de estos elementos se obtendrá que, con un $2,7 \%$ en peso de litio ${ }^{[2]}$ (máximo valor de solubilidad según diagrama de equilibrio) (Fig. 1), la solución tendría una densidad de $6.270 \mathrm{~kg} / \mathrm{m}^{3}$, es decir, teóricamente sería un 29,7 \% más ligera que el cobre puro. Esta modificación en la densidad teórica es una de las motivaciones para encontrar métodos alternativos de desarrollo para estas aleaciones.

En los últimos años se han realizado trabajos tendientes a desarrollar aleaciones $\mathrm{Cu}$-Li mediante nuevos procesos que no involucren la etapa de fusión. En este sentido, Peñaloza et al..$^{[3-8]}$ han trabajado en la obtención de estas aleaciones mediante el proceso de electrodeposición. De acuerdo a los resultados presentados por estos investigadores, mediante este proceso ha sido posible fabricar aleaciones con un contenido atómico de litio cercano al máximo en equilibrio, 2,7 $\%$ en peso a $453,5 \mathrm{~K}$, pero a temperatura ambiente (Fig. 1). En el presente trabajo se ha estudiado la alternativa de producir estas aleaciones mediante aleado mecánico (AM), proceso mediante el cual se lleva a cabo la combinación o aleación de dos o más elementos y en el cual se emplean medios mecánicos de deformación. Cuando se desea modificar microestructuralmente un material y no realizar aleaciones por medios mecánicos, el proceso se denomina molienda mecánica (MM). Una de las principales ventajas en el desarrollo de aleaciones sobresaturadas o con solubilidad extendida por aleado mecánico es que, cuando se trata de elementos con temperaturas de fusión muy diferentes, se evita la fusión ya que la aleación se genera por transformaciones en estado sólido. De acuerdo a los antecedentes investigados ${ }^{[9-13]}$, prácticamente no existen estudios del comportamiento de cobre y litio sometidos a la molienda de alta energía. Sin embargo, se ha estudiado que aleaciones con elementos que presentan una solubilidad en estado sólido limitada

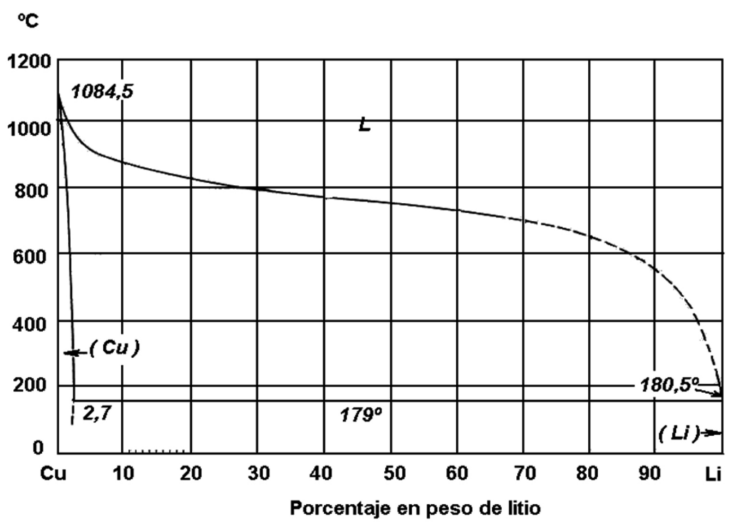

Figura 1.Diagrama de fases $\mathrm{Cu}-\mathrm{Li}^{[2]}$.

Figure 1. The Cu-Li phase diagram ${ }^{[2]}$. en el cobre, pueden fabricarse mediante este proceso, con un rango de solubilidad más amplio o extendido, comparado con respecto a métodos convencionales. Los primeros resultados obtenidos por los autores de este grupo de investigación han mostrado que, efectivamente, el desarrollo de estas aleaciones por AM es posible. Sin embargo, los parámetros óptimos del proceso se encuentran aún en etapa de evaluación. La atmósfera de control es uno de estos parámetros y el presente trabajo muestra nuestros resultados al evaluar el efecto de las atmósferas argón y nitrógeno sobre la molienda de una mezcla cobre con $2,5 \%$ en peso de litio y la de cobre puro.

\section{MATERIALES Y PROCEDIMIENTO EXPERIMENTAL}

El proceso se realizó en un molino SPEX 8000D con una velocidad nominal de $1.500 \mathrm{rpm}$, empleando contenedores y bolas de acero del mismo material. La razón en peso bolas polvo (RBP) utilizada en todas las moliendas fue de 10:1. La carga de los contenedores se realizó en el interior de una cámara, que fue llenada con argón o nitrógeno. Los tiempos de molienda empleados fueron desde 3 a $30 \mathrm{~h}$. Para las moliendas de cobre y litio, cada contenedor se cargó con $5 \mathrm{~g}$ de polvo, 97,5\% en peso de cobre (pureza 99,7 \% en peso, Merck) y 2,5\% en peso de litio (pureza $>99 \%$ en peso, Merck). Tanto en las moliendas de $\mathrm{Cu}-\mathrm{Li}$ como en las de cobre puro, no se emplearon agentes de control. Los polvos procedentes de la molienda se analizaron mediante diversas técnicas. La difracción de rayos X (DRX) se realizó en un equipo Siemens 5000 con radiación $\mathrm{K} \alpha$ de $\mathrm{Cu}$, barriendo desde $2 \theta$ (grados) $=20$ hasta $100^{\circ}$. La espectroscopía de absorción atómica (EAA) para la determinación de litio y hierro se realizó en un equipo GBS 905. Para la determinación de oxígeno se empleó un equipo LECO, modelo TC-436, el cual realiza la cuantificación mediante espectroscopía infrarroja. Las observaciones a nivel microestructural fueron realizadas en un microscopio FEG SEM Philips, XL 30.

\section{RESULTADOSY DISCUSION}

\subsection{Moliendas de $\mathrm{Cu}-\mathrm{Li}$ realizadas con argón como atmósfera de control}

\subsubsection{Difracción de rayos $X$ : Efectos del $A M$}

Cuando se producen aleaciones a partir de elementos puros mediante aleado mecánico se considera que la 
INFLUENCIA DE LA ATMÓSFERA DE CONTROL Y TIEMPO DE MOLIENDA SOBRE LA MORFOLOGÍA Y MICROESTRUCTURA DE POLVOS DE COBRE PURO Y COBRE-2,5 \% LITIO... INFLUENCE OF THE CONTROL ATMOSPHERE AND MILLING TIME ON THE MORPHOLOGY AND MICROSTRUCTURE OF PURE COPPER AND COPPER-2.5 \% LITHIUM POWDERS...

disolución se ha realizado de manera efectiva, cuando no es posible detectar los picos de difracción del soluto ${ }^{[9]}$. Dadas las condiciones de difracción y de los elementos en estudio, sólo fue posible estudiar la difracción de los planos (111), (200), (220), (311) y (222) del cobre, las que según el archivo de polvos $\mathrm{PDF}$, se presentan en $2 \mathrm{q}=43,295^{\circ} ; 50,431^{\circ} ; 74,127^{\circ}$; $89,926^{\circ}$ y $95,135^{\circ}$, respectivamente.

De acuerdo a los resultados, los picos de difracción correspondientes a los planos antes mencionados, mostraron cambios a medida que se incrementó el tiempo de molienda. En la tabla I, las columnas segunda y tercera muestran los ángulos en los cuales se produjo la difracción de los planos (111) y (200) del cobre. En estos resultados se observa que, hasta $24 \mathrm{~h}$, los ángulos fueron desplazándose hacia menores ángulos, comportamiento asociado al incremento del parámetro de red o a la presencia de maclas y fallas de apilamiento. Normalmente, se asume como la formación de una solución sólida con un soluto de mayor tamaño atómico que el solvente. En el caso del sistema en estudio, el cobre posee un radio atómico de $1,45 \mathrm{~A}^{0}$, que es inferior a los $1,67 \mathrm{~A}^{0}$ del litio, por lo que, efectivamente, de existir solución sólida debería producirse un incremento en el parámetro de red ${ }^{[9]}$. Con $30 \mathrm{~h}$ de molienda, al extraer los polvos del contenedor, éstos se encontraban aparentemente oxidados pues su color era distinto al observado en las demás muestras. El ensayo se repitió dos veces y, en todos ellos, se observó el mismo color en los polvos. Tras hacer los análisis de DRX y debido al intenso grado de deformación del material en general, la información que se pudo extraer de los diagramas de difracción no fue concluyente en términos de la presencia de óxidos de litio. Sin embargo, dado que los picos de difracción de los planos de cobre estuvieron menos desplazados hacia ángulos menores, es posible suponer que parte del litio se combinó químicamente con oxígeno y dejó de formar parte de la solución sólida. Este tipo de reacciones ha sido mostrada en otros sistemas donde los elementos se encuentran formando soluciones sobresaturadas y, posteriormente, forman otro tipo de compuesto dependiendo de las condiciones y atmósfera de molienda ${ }^{[9]}$.

El proceso de aleado mecánico también promueve el refinamiento de las partículas de polvo y del tamaño de cristalito de los elementos o compuestos empleados como materias primas, mientras incrementa las tensiones internas. Los efectos del proceso promueven el ensanchamiento de los picos de difracción pero no inciden en el desplazamiento de los mismos, sin embargo, pueden dificultar su localización. De este modo, de los efectos promovidos por el proceso, el único que explica el desplazamiento de los ángulos es la formación de una solución sólida con un soluto de mayor tamaño atómico. Sin embargo, el litio no fue el único elemento presente en las muestras después de realizadas las moliendas; cómo se verá más adelante. También, fue detectado hierro, el cual se incorpora a los polvos como un contaminante procedente del contenedor y las bolas de molienda. El hierro presenta una solubilidad máxima en cobre del $4 \%$ en peso y un radio atómico de $1,56 \mathrm{~A}^{0}$, razón por la cual es posible que, este elemento, también se encuentre en solución sólida y promoviendo un efecto muy similar al del litio en el desplazamiento de los picos de difracción hacia ángulos más pequeños. En este sentido, la única forma de corroborar que el efecto es por el litio y no por el hierro consistió en moler cobre puro bajo las mismas condiciones que la mezcla $\mathrm{Cu}-2,5 \%$ peso Li, medir el contenido de hierro en ambos casos y estudiar los desplazamientos. Como se verá más adelante, los análisis químicos de las muestras tras la molienda de cobre puro mostraron hierro, pero los desplazamientos no fueron los observados en $\mathrm{Cu}-\mathrm{Li}$, incluso cuando se realizaron las moliendas en nitrógeno (Tabla II).

Como se mencionó anteriormente y debido a que el refinamiento microestructural es otra consecuencia del proceso de aleado mecánico, se emplearon los resultados de DRX para estimar el tamaño de cristalito del cobre tras la molienda aplicando el método de

Tabla I. Resultados de los análisis de DRX de las muestras de Cu+Li molidas usando argón

Table I. XRD results of $\mathrm{Cu}+$ Li samples milled using argon

\begin{tabular}{cccccc}
\hline $\begin{array}{c}\text { Tiempo de } \\
\text { molienda (h) }\end{array}$ & Plano (111) Cu & $\begin{array}{c}\mathbf{2}^{\circ} \text { (grados) } \\
\text { Plano }(\mathbf{2 0 0}) \mathbf{C u}\end{array}$ & $\begin{array}{c}\text { Tamaño de } \\
\text { cristalito }(\mathbf{n m})\end{array}$ & $\begin{array}{c}\text { Parámetro } \\
\text { de red }(\mathbf{n m})\end{array}$ & $\begin{array}{c}\text { Dureza } \\
\text { HV }\end{array}$ \\
\hline 6 & 43,337 & 50,364 & 20 & 0,3621 & 257 \\
12 & 43,205 & 50,353 & 18 & 0,3623 & 321 \\
18 & 43,218 & 50,242 & 15 & 0,3624 & 362 \\
24 & 43,186 & 50,202 & 13 & 0,3625 & 357 \\
30 & 43,313 & 50,362 & 15 & 0,3618 & 257 \\
\hline
\end{tabular}


Tabla II. Resultados de los análisis de DRX de las muestras $\mathrm{Cu}$ y Cu+Li, molidas usando nitrógeno Table II. XRD results of samples $\mathrm{Cu}$ and $\mathrm{Cu}+\mathrm{Li}$, milled using nitrogen

\begin{tabular}{ccccccc}
\hline $\begin{array}{c}\text { Tiempo de } \\
\text { molienda } \\
\text { (h) }\end{array}$ & $\begin{array}{c}2 \theta^{\circ} \text { (grados) } \\
\text { Plano (111) } \\
\text { Cu }\end{array}$ & $\begin{array}{c}2 \theta^{\circ} \text { (grados) } \\
\text { Plano (111) } \\
\text { (Cu+Li) }\end{array}$ & $\begin{array}{c}2 \theta^{\circ} \text { (grados) } \\
\text { Plano (200) } \\
\text { del Cu }\end{array}$ & $\begin{array}{c}2 \theta^{\circ} \text { (grados) } \\
\text { Plano (200) } \\
\text { del (Cu+Li) }\end{array}$ & $\begin{array}{c}2 \theta^{\circ} \text { (grados) } \\
\text { Plano (220) } \\
\text { del Cu }\end{array}$ & $\begin{array}{c}2 \theta^{\circ} \text { (grados) } \\
\text { Plano (220) } \\
\text { del (Cu+Li) }\end{array}$ \\
\hline 0 & 43,468 & 43,468 & 50,596 & 50,596 & 74,294 & 74,294 \\
3 & 43,474 & 43,329 & 50,544 & 50,315 & 74,241 & 74,076 \\
6 & 43,495 & 43,396 & 50,561 & 50,346 & 74,258 & 74,135 \\
12 & 43,527 & 43,451 & 50,596 & 50,424 & 74,26 & 74,142 \\
24 & 43,632 & 43,596 & 50,707 & 50,566 & 74,338 & 74,217 \\
\hline
\end{tabular}

Williamson-Hall ${ }^{[14]}$. Según los cálculos realizados (Tabla I), el tamaño de cristalita del cobre resultó de rango nanométrico, incluso después de periodos de molienda relativamente cortos. En trabajos publicados anteriormente ${ }^{[15 \text { y } 16]}$ se ha mostrado que el tamaño de cristalito del cobre puede alcanzar dimensiones nanométricas mediante aleado mecánico, pero, según dichos trabajos, los tiempos de molienda requeridos fueron algo superiores a los obtenidos en el presente trabajo. Los trabajos citados, no se realizaron con el mismo soluto con el qur se realizó este estudio, pero si bajo las mismas condiciones de molienda, es decir, misma velocidad y RBP, lo que permite suponer que fue, precisamente, el litio quien facilitó el refinamiento. Una posible explicación es suponer que, si el litio entra en solución dentro del cobre promoverá un endurecimiento por solución sólida lo que, junto al endurecimiento por deformación, facilitará los eventos de fractura que conllevan al refinamiento de las partículas. En la tabla I se muestra que efectivamente la dureza de los polvos se incrementó con el tiempo de molienda.

\subsubsection{Espectroscopía de absorción atómica: contenido de hierro}

Debido a que el aleado mecánico promueve el endurecimiento por deformación de materiales, éstos pueden promover el desgaste del material de los contenedores y bolas. Si estos últimos son de acero, el desgaste promoverá la contaminación del material molido, principalmente, con hierro ${ }^{[9-11}$ y 17$]$. Por ello, se analizaron mediante espectroscopía de absorción atómica para determinación del contenido de hierro las muestras procedentes de las moliendas de $\mathrm{Cu}$ 2,5 \% peso Li, realizadas en argón. Los resultados obtenidos se presentan en la figura 2 y reflejan que las muestras contienen hierro y que su cantidad es una

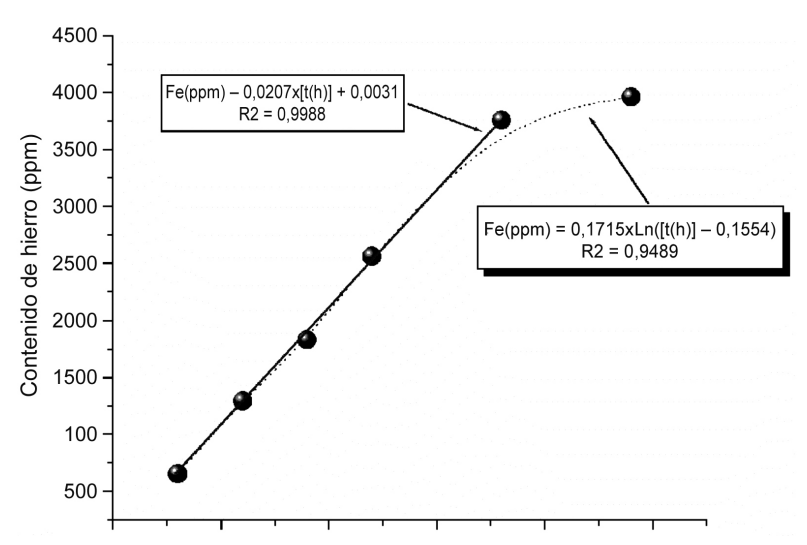

Figura 2. Contaminación por hierro en los polvos de Cu-2,5 \% Li en función del tiempo de molienda (argón).

Figure 2. Iron contamination in Cu-2.5\% Li powders as a function of milling time (argon).

función del tiempo de molienda. Analizando los valores obtenidos se observó un comportamiento de tipo lineal entre el contenido de hierro (ppm) y el tiempo de molienda (h) hasta las $18 \mathrm{~h}$, (Ec. (1)). La calidad del ajuste de los puntos con la ecuación (1) se manifiesta en el coeficiente de determinación $\mathrm{R}^{2}$, el cual muestra lo ajustada que está la nube de puntos a la línea de regresión. Después de las 18 h se apreció un cambio en la tendencia de los puntos con un comportamiento asintótico al 0,4\% en peso de hierro y la regresión, claramente, ya no fue lineal, obteniéndose un $\mathrm{R}^{2}$ menor a 0,9. Dado que el coeficiente de determinación no fue satisfactorio se buscó otra función que ajustase adecuadamente todos los puntos hasta 24 h. La ecuación resultante no fue lineal sino que representó un comportamiento asintótico representado a través de la ecuación (2), la cual posee un coeficiente de determinación superior a 0,9. Este comportamiento podría justificarse con el hecho de 
INFLUENCIA DE LA ATMÓSFERA DE CONTROL Y TIEMPO DE MOLIENDA SOBRE LA MORFOLOGÍA Y MICROESTRUCTURA DE POLVOS DE COBRE PURO Y COBRE-2,5 \% LITIO... INFLUENCE OF THE CONTROL ATMOSPHERE AND MILLING TIME ON THE MORPHOLOGY AND MICROSTRUCTURE OF PURE COPPER AND COPPER-2.5 \% LITHIUM POWDERS...

que, efectivamente, tras $18 \mathrm{~h}$, la mezcla cobre + litioi, no continúa endureciéndose sino que mantiene su dureza, lo que podría explicar el contenido constante en hierro.

$$
\begin{gathered}
\mathrm{Fe}(\mathrm{ppm})=0,0207 \cdot[\mathrm{t}(\mathrm{h})]+0,0031 \\
\mathrm{R} 2=0,9988 \text { Para } \mathrm{t} \leq 18 \mathrm{~h}
\end{gathered}
$$

$$
\begin{aligned}
& \mathrm{Fe}(\mathrm{ppm})=0,1715 \cdot \mathrm{Ln}([\mathrm{t}(\mathrm{h})]-0,1554 \\
& \mathrm{R}^{2}=0,9489 \text { Para } 18 \mathrm{~h} \leq \mathrm{t} \leq 24 \text { horas }
\end{aligned}
$$

El refinamiento microestructural promovido por el AM se encuentra bastante documentado, como también el hecho de que algunos elementos sometidos a este proceso alcanzan tamaños de cristalita mínimos. Un ejemplo es el trabajo realizado en molibdeno por Lucks et al. ${ }^{[17]}$. Esos autores observaron que tras $10 \mathrm{~h}$ de molienda el refinamiento cambiaba drásticamente su tasa de avance haciéndose, prácticamente, estacionario después de $80 \mathrm{~h}$ de molienda, bajo argón en un molino atritor. De esta manera, los resultados obtenidos en este estudio señalarían que es el grado de refinamiento que alcanza el material molido lo que determina el nivel de contaminación por hierro. Lo anterior puede ser corroborado con los datos de dureza obtenidos (Tabla I).

\subsection{Moliendas de Cu-Li y cobre puro, realizadas con nitrógeno como atmósfera de control}

El proceso de AM se realiza, normalmente, en atmósferas inertes con el objetivo de minimizar y/o prevenir la oxidación y/o contaminación de los polvos molidos $^{[9,10}$ y 17$]$. Pese a lo anterior, ambos fenómenos son muy frecuentes en materiales obtenidos mediante AM y, en el caso de la oxidación, cuando en las materias primas se emplean materiales con elevada afinidad por el oxígeno como son el caso del titanio, magnesio y aluminio, el efecto es aún mayor ${ }^{[18]}$. Aunque resulte extraño, pocos trabajos realizados en AM han reflejado los contenidos de oxígeno; de hecho, la mayoría de los trabajos en los que se muestra la composición del material obtenido tras la molienda son los referidos a molienda criogénica y pertenecientes al grupo de Lavernia et al. ${ }^{[19-22]}$. Debido a que no existen datos disponibles en la literatura acerca de los contenidos de oxígeno en el cobre puro sometido a AM, se consideró necesario obtener estos datos, moliendo, por un lado, cobre puro y, por otro, cobre con 2,5\% en peso de litio, bajo las mismas condiciones de molienda, para comparar el efecto del litio en la incorporación de oxígeno. Para que es- te efecto fuese más notorio, se sustituyó la atmósfera de argón $(<2 \mathrm{ppm} \mathrm{O})$ por nitrógeno $(<10 \mathrm{ppm} \mathrm{O})$.

\subsubsection{Difracción de rayos $X$ : Efectos del $A M$}

Los resultados de DRX mostraron, claramente, dos discrepancias entre los patrones de las muestras de $\mathrm{Cu}$ y $\mathrm{Cu}$-Li molidas en nitrógeno: el ancho y la posición de los picos de difracción de los planos del cobre.

En la figura 3 se comparan los anchos de los picos de difracción de los planos (111) y (200) del Cu después de la molienda y se observa, claramente, el mayor ensanchamiento promovido en las muestras $\mathrm{Cu}-\mathrm{Li}$ con respecto a las de Cu puro y además, se observa que dicha diferencia se hace mayor en función del tiempo de molienda. El ensanchamiento y la pérdida de intensidad de los picos de difracción en función del tiempo de molienda son, tal y como se ha explicado, respuestas normales de los materiales frente al proceso de AM y son consecuencia de los fuertes cambios microestructurales inducidos por la intensa deformación. La diferencia entre los dos materiales aquí analizados, un 2,5\% en peso de litio, de entrar en solución sólida como se ha supuesto, alteraría las propiedades del cobre, otorgándole una respuesta diferente frente a las condiciones de molienda, respuesta que, teóricamente, debería ser de menor ductilidad que la del cobre puro, pues la incorporación de litio promovería un endurecimiento por solución sólida. Como se ha señalado anteriormente, para comprobar que el litio se encuentra efectivamente en solución sólida, es necesario que los DRX muestren cambios en el parámetro de red

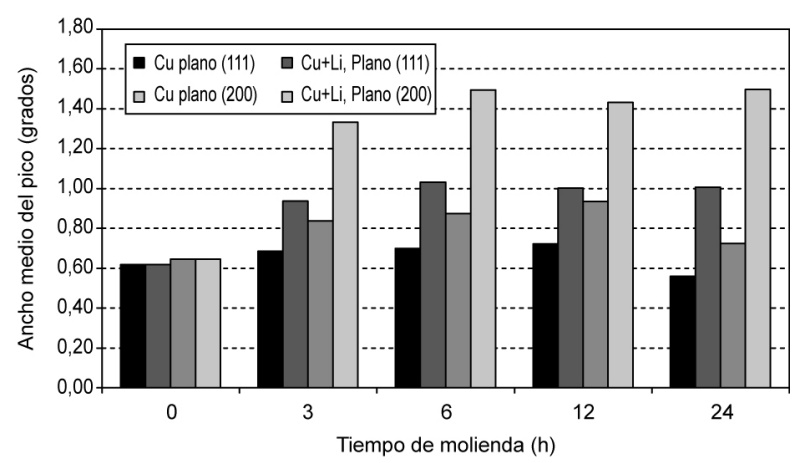

Figura 3. Anchos de picos de difracción de rayos $X$ de los planos (111) y (200) del cobre molido con y sin litio (nitrógeno).

Figure 3. XRD peak width of the (111) and (200) planes of copper milled with and without lithium (nitrogen). 
del cobre. En la tabla II se muestran las diferencias encontradas en las posiciones de difracción de los planos (111) y (200) del cobre tras la molienda, en las muestras de $\mathrm{Cu}$ y $\mathrm{Cu}-\mathrm{Li}$, advirtiéndose claramente que cuando se realiza la molienda con litio, las posiciones de los planos de difracción después de la molienda son siempre menores a las del cobre, corroborando la hipótesis que, mediante el proceso, este elemento estaría siendo incorporado como soluto en el cobre.

\subsubsection{Espectroscopía: contenidos de oxígeno y hierro}

El oxígeno es una impureza presente en materiales sometidos a AM asociada a la atmósfera de molienda y cuyo contenido se incrementa en función del tiempo ${ }^{[9]}$. Adicionalmente, existen antecedentes que señalan que, cuando materiales con elevada afinidad por el oxígeno, como el titanio, son sometidos a molienda, las cantidades de este elemento no metálico también se elevan en las mezclas finales ${ }^{[18]}$. Los resultados de este estudio, representados en la figura 4, muestran claramente que, cuando el cobre es molido sólo, el contenido de oxígeno se incrementa con el tiempo. Sin embargo, cuando es molido con un 2,5\% en peso de litio, el contenido de oxígeno se incrementa hasta cinco veces, para un mismo tiempo de molienda. El elevado contenido de oxígeno en los polvos de $\mathrm{Cu}-2,5 \%$ en peso de litio demuestra la importancia de este elemento como contaminante en el proceso, según lo sostenido por Suryanarayana ${ }^{[18]}$ y, al mismo tiempo, pone de manifiesto una limitación

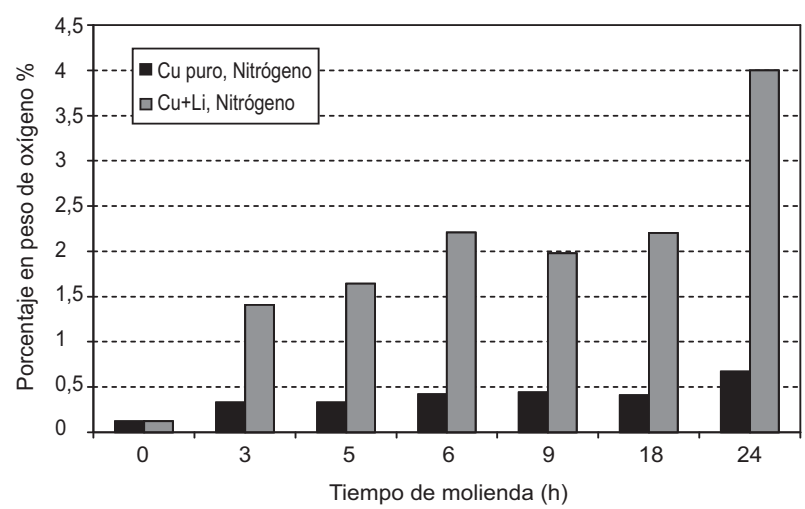

Figura 4. Contaminación de oxígeno en los polvos de Cu y Cu-2,5\% Li en función del tiempo de molienda (nitrógeno).

Figure 4. Oxygen contamination in $\mathrm{Cu}$ and $\mathrm{Cu}$ $2.5 \%$ Li powders as a function of milling time (nitrogen). para el proceso de consolidación. En este sentido, cabe destacar que no se detectaron picos de difracción correspondientes a óxidos en los difractogramas; sin embargo, este hecho no puede ser interpretado como una evidencia irrefutable de que estas fases no se encuentren presentes, sino más bien de que su contenido no es suficiente para el límite de detección de dicha técnica.

En cuanto a la solubilidad del oxígeno en cobre se debe considerar que el soluto, en este caso, es un elemento no metálico y, como tal, presentará solubilidad sólida muy restringida en cobre y tenderá a formar los compuestos $\mathrm{CuO}$ y $\mathrm{Cu}_{2} \mathrm{O}$ antes que la solución sólida. En efecto, la solubilidad máxima de oxígeno en cobre se alcanza a los $1.339 \mathrm{~K}$ y es de $0,0035 \%$ en peso ${ }^{[2]}$.

En el caso de las moliendas con cobre y litio, y debido a la elevada afinidad química entre litio y oxígeno, es probable que el oxígeno se encuentre combinado con el litio; sin embargo, la cantidad de óxidos formados es pequeña, por cuanto los análisis realizados mediante DRX no detectarían la presencia de los óxidos.

En cuanto a los contenidos de hierro en las muestras molidas en presencia de nitrógeno, la figura 5 muestra las diferencias encontradas con las moliendas realizadas en argón. De acuerdo a estos resultados, es claro que las muestras de $\mathrm{Cu}-2,5 \%$ en peso de litio presentaron un comportamiento relativamente similar siendo, en ambos casos, la cantidad de hierro una función del tiempo de molienda y alcanzando valores máximos cercanos a $0,4 \%$ en peso de hierro. Los mayores contenidos de hierro fueron encontrados en las muestras de cobre puro molido en

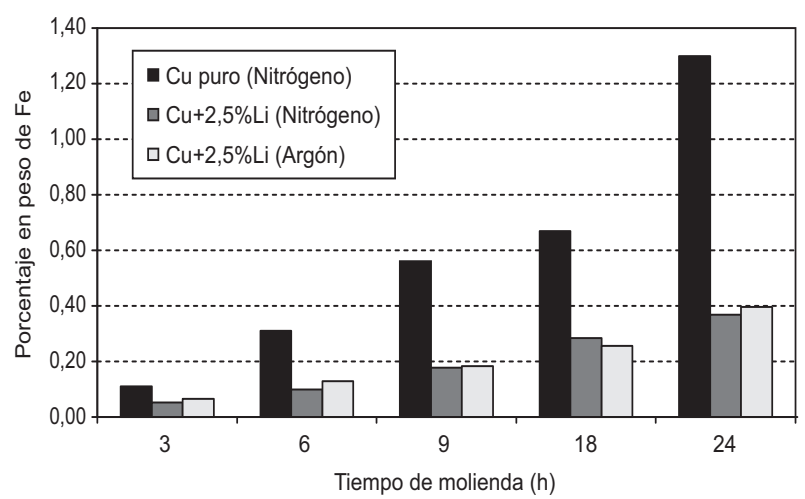

Figura 5. Contaminación por hierro en los polvos Cu y Cu-2,5 \% Li en función del tiempo de molienda (nitrógeno y argón).

Figure 5. Iron contamination in $\mathrm{Cu}$ and $\mathrm{Cu}-2.5$ $\%$ Li powders as a function of milling time (nitrogen and argon). 
INFLUENCIA DE LA ATMÓSFERA DE CONTROL Y TIEMPO DE MOLIENDA SOBRE LA MORFOLOGÍA Y MICROESTRUCTURA DE POLVOS DE COBRE PURO Y COBRE-2,5 \% LITIO... INFLUENCE OF THE CONTROL ATMOSPHERE AND MILLING TIME ON THE MORPHOLOGY AND MICROSTRUCTURE OF PURE COPPER AND COPPER-2.5 \% LITHIUM POWDERS...

nitrógeno y superaron el 1,2 \% a $24 \mathrm{~h}$. La explicación a estos resultados se atribuyó a las diferencias morfológicas encontradas, que serán descritas a continuación.

\subsubsection{Microscopía electrónica de barrido: morfología de las partículas}

Además de las diferencias en sus contenidos de oxígeno y hierro, las muestras $\mathrm{Cu}$ y $\mathrm{Cu}-\mathrm{Li}$ mostraron

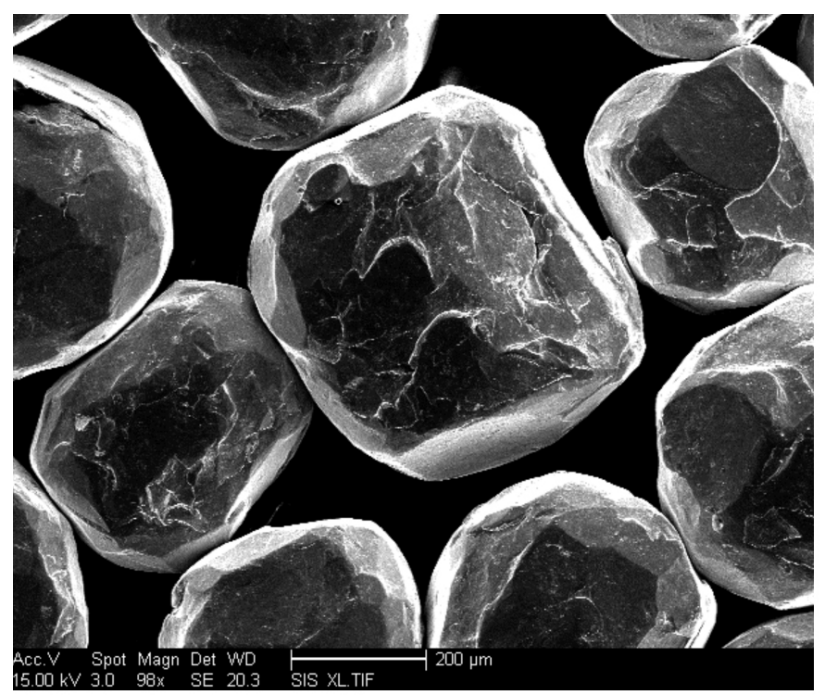

a)

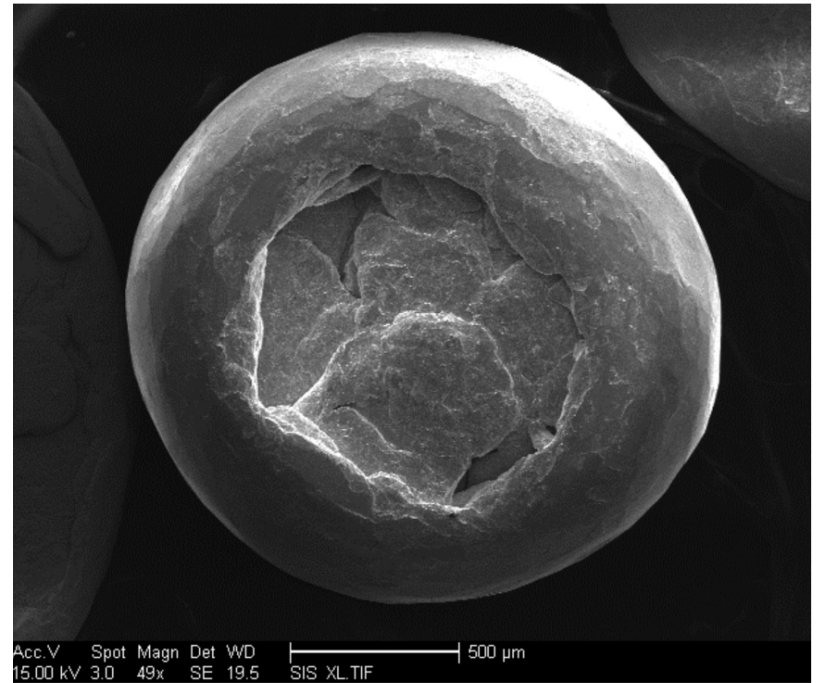

c) grandes diferencias morfológicas después de la molienda. El resultado más notable fue el del cobre puro (Fig. 6), que, paulatinamente, fue formando esferas hasta alcanzar dimensiones milimétricas. Varios autores han señalado que para evitar la formación de estas esferas deben usarse agentes de control ${ }^{[9-11]}$, los cuales no fueron empleados en las experiencias realizadas. Pero, al comparar los resultados obtenidos con cobre puro y cobre con litio, se advierte que la presencia de litio genera un efecto similar al de un agente de control, evitando la formación de esferas

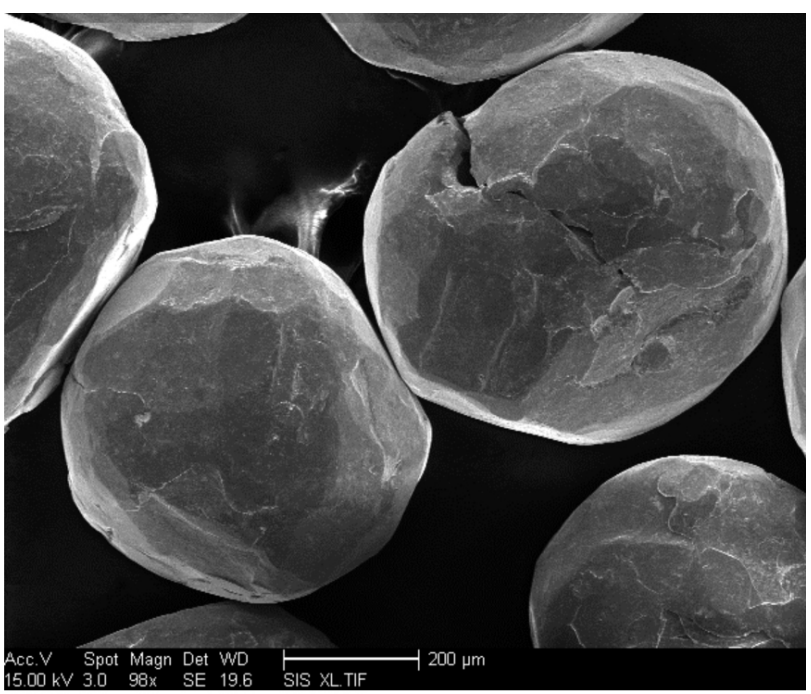

b)

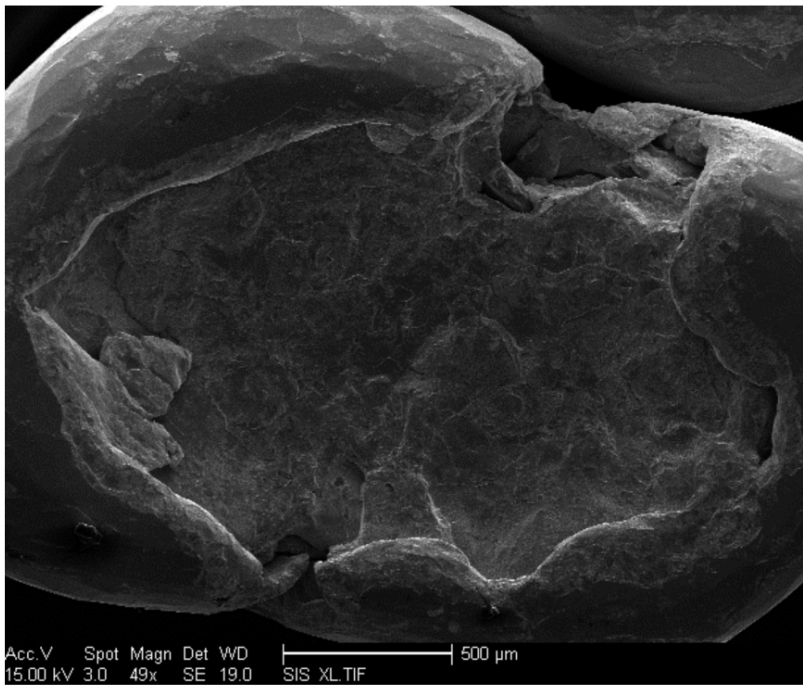

d)

Figura 6. Micrografías electrónicas de barrido en las que se muestran los cambios microestructurales del Cu puro (nitrógeno) con el aleado mecánico: a) 3 h, 100x; b) 9 h, 100x; c) 18 h, 50X; y (d) 24 h, $50 x$.

Figure 6. SEM micrographs showing the changes in morphology of mechanically-milled pure $\mathrm{Cu}$ powders (nitrogen): a) $3 h, 100 x$; b) $9 h, 100 x$; c) $18 h, 50 x$; $y$ d) $24 h, 50 x$. 
(Fig. 7). Dada la morfología de estos aglomerados y la continua soldadura y fractura producidas durante el proceso, la granulometría de estas muestras se encuentran entre un gran rango de tamaños de partícula, que va desde las unidades a los cientos de micras.

De las micrografías obtenidas se desprende que, cuando el cobre es molido mecánicamente, bajo las mismas condiciones de molienda que el cobre $+2,5$ $\%$ en peso de litio, es decir, en el mismo molino, sin agentes de control, bajo la misma atmósfera y con la misma RBP; se formarán esferas que crecerán en función del tiempo de aleado y que, para tiempos mayores a $3 \mathrm{~h}$, tendrán tamaño suficiente para generar

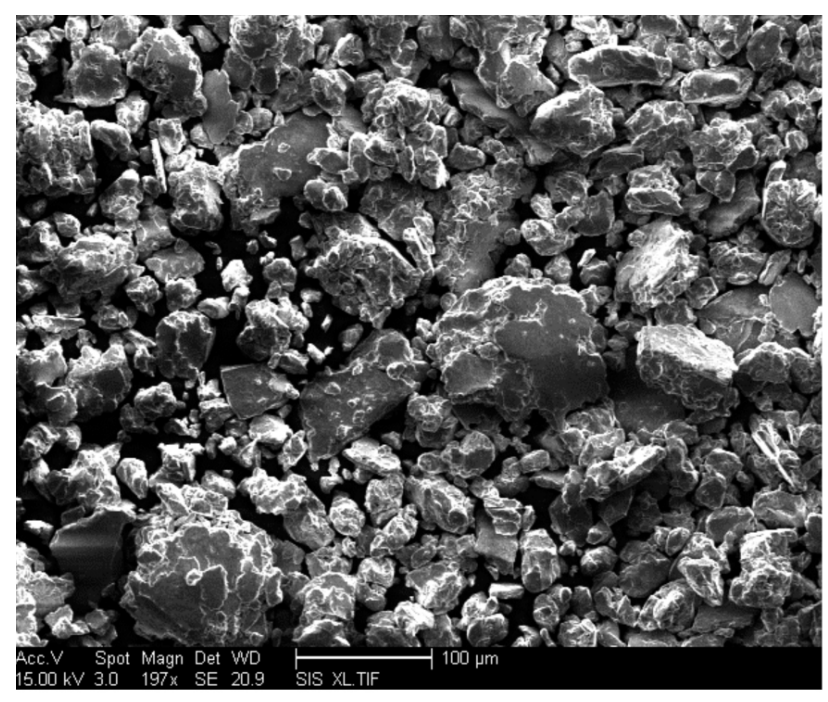

a)

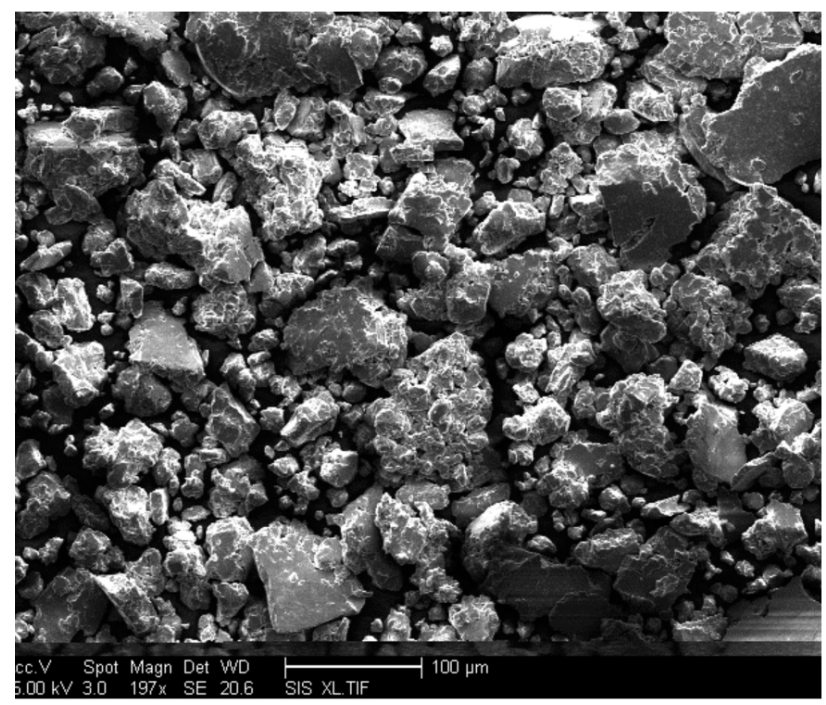

c) un efecto fuertemente abrasivo sobre los contenedores de acero. El efecto será mayor a medida que el radio de las esferas se incremente, lo que efectivamente ocurrió en función del tiempo de molienda. A mayor radio de las esferas de cobre, mayor será la abrasión y, como consecuencia, mayor la contaminación por hierro, como se demostró en los resultados.

\section{CONCLUSIONES}

- Bajo un adecuado control de los parámetros del proceso de AM, es posible la obtención de

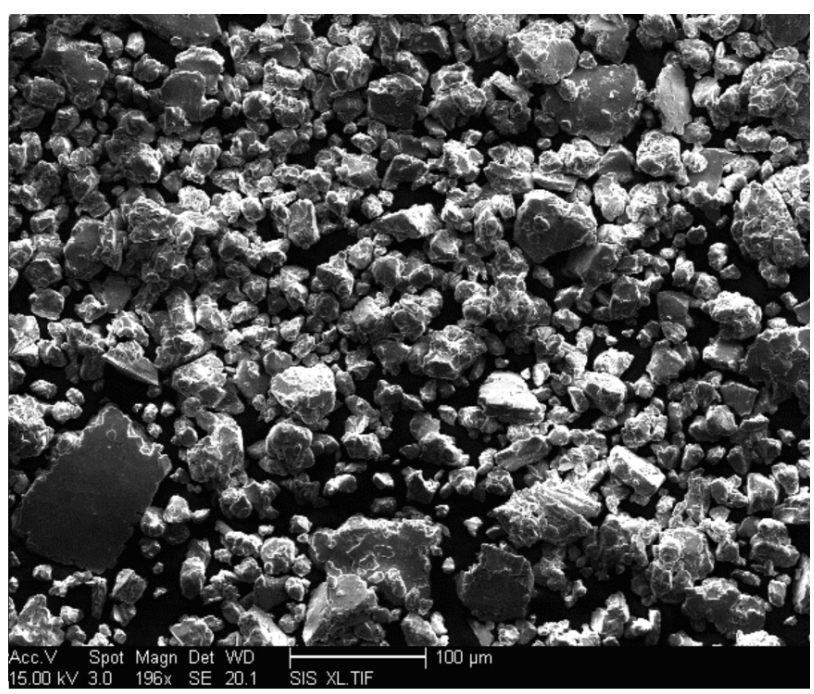

b)

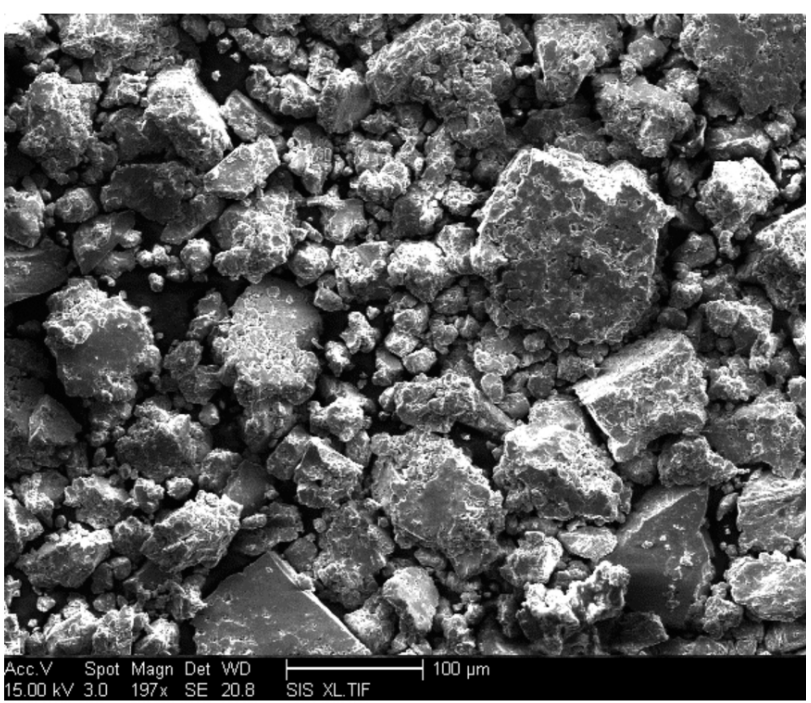

d)

Figura 7. Micrografías electrónicas de barrido en las que se muestran los cambios morfológicos, en polvos de Cu-2,5 \% Li con aleado mecánico (nitrógeno, 200 x): a) 3 h; b) 9 h; c) 18 h; y d) 24 h.

Figure 7. SEM micrographs showing the changes in morphology of mechanically-alloyed $\mathrm{Cu}-2.5 \%$ Li powders (nitrogen, 200x): a) $3 h$; b) $9 h$; c) $18 h$; yd) $24 h$. 
INFLUENCIA DE LA ATMÓSFERA DE CONTROL Y TIEMPO DE MOLIENDA SOBRE LA MORFOLOGÍA Y MICROESTRUCTURA DE POLVOS DE COBRE PURO Y COBRE-2,5 \% LITIO... INFLUENCE OF THE CONTROL ATMOSPHERE AND MILLING TIME ON THE MORPHOLOGY AND MICROSTRUCTURE OF PURE COPPER AND COPPER-2.5 \% LITHIUM POWDERS...

soluciones sólidas supersaturadas de litio en cobre a temperatura ambiente.

- Al emplear argón o nitrógeno como atmósfera de control, no se modifican los tiempos de molienda requeridos para la formación de la solución sólida, sin embargo, se alteran los contenidos de oxígeno, siendo mayores en las muestras molidas en nitrógeno.

- Si el cobre es molido en forma pura, el proceso de molienda promueve la formación de hojuelas, las que, a su vez, se van soldando y generan esferas que crecen gracias a las sucesivas soldaduras llegando a tamaños milimétricos.

- Las esferas de cobre actúan como medios de abrasión de los contenedores de acero y dado que, a mayor tiempo de molienda, mayor fue el radio de las esferas, mayor fue la contaminación por hierro.

- Durante la molienda de cobre y litio, la presencia de éste último elemento modifica la respuesta del cobre frente al proceso. Las modificaciones promovidas, por el litio al cobre, se manifiestan en cambios microestructurales, similares a los observados al emplear agentes de control y en los contenidos de oxígeno y hierro.

- Además de los cambios morfológicos presentados por las muestras con y sin litio, se detectaron cambios en los contenidos de oxígeno, elemento que se encontró en mayor cantidad en las muestras con litio y con mayores tiempos de molienda, efecto que está propiciado por la presencia de litio.

\section{Agradecimientos}

Los autores de este trabajo desean manifestar su agradecimiento al apoyo prestado por la Dirección de Investigación de la Pontificia Universidad Católica de Valparaíso y del Programa Bicentenario de Ciencia y Tecnología, PSD-06, del Gobierno de Chile.

\section{REFERENCIAS}

[1] C. Brooks, Heat treatment structure and properties of nonferrous alloys, ASM, Metals Park, Ohio 44073, EE. UU. 1982, pp. 275-285.

[2] Asm Handbook Committee, Metal Handbook vol. 8, $8^{\text {th }}$ edition, ASM, Metals Park, Ohio 44073, EE. UU., pp. 294.

[3] A. Peñaloza, M. Ortiz y Ch. Wörner, J. Mater. Lett. 14 (1995) 511-513.
[4] O. Lambri, A. Peñaloza, A. Morón, M. Ortíz y F. Luca, Mater. Sci. Eng. A 212 (1996) 108-118.

[5] O. Lambri, A. Morón, G. Lambri, A. Peñaloza, M. Ortiz, C. Wörner y E. Bocanegra, Mater. T. JIM. 40 (1999) 72-77.

[6] O. Lambri, J.I. Pérez-Landazábal, A. Peñaloza, O. Herrero, V. Recarte, M. Ortiz y C.H. Wörner, Mater. Res.. Bull. 35 (2000) 1.023. 1.033.

[7] P. Diaz, C. Wörner, C. Carrasco, M. Ortiz y A. Peñaloza, Rev. Metal. Madrid 41 (2005) 323. 329

[8] J.I.Pérez-Landazábal, O. Lambri, A. Peñaloza, V. Recarte, J. Campo, L. Salvatierra, O. Herreo, M. Ortiz, L.M. Milani y C.H. Wörner, J. Mater. Lett. 56 (2002) 709-715.

[9] C. Suryanarayana, Prog. Mater. Sci. 46 (2001) $1-184$.

[10] L. Lu y M-O. Lai, Mechanical Alloying, Kluwer Academic Publishers, EE. UU., 1998, pp. 23. 65 .

[11] P.R. Soni, Mechanical Alloying, Cambridge International Science Publishing, Inglaterra, 2000, pp. 15-23

[12] R. Cahn y P. Haasen, Physical Metallurgy, Elsevier Science, The Netherlands, 1996, pp. 1.724-1.818.

[13] P.A. Rojas, A. Peñaloza, C.H. Wörner, R. Fernández y A. Zúñiga. J. Alloy. Compd. 425 (2006) 334-338.

[14] B. Cullity y S. Stock, Elements of X-Ray Diffraction, Prentice Hall, Upper Saddle River, NJ 07458, EE. UU., 2001, pp 170-173.

[15] T. Raghu; R. Sundaresan, P. Ramakrishnan y T. Rama, Mater. Sci. Eng. A 304-306 (2001) 438-441.

[16] E. Botcharova, J. Freudenbergger, L. Schultz, J. Alloy. Compd. 365 (2004) 157-163.

[17] I. Lucks, P. Lamparter y E.J. Mittemeijer, Acta Mater. 49 (2001) 2.419-2.428.

[18] C. Suryanarayana, Mechanical Alloying and Milling Marcel Dekker, EE. UU., 2004 pp. 59. 78.

[19] F. Zhou, X.Z. Liao, Y.T. Zhu, S. Dallek y E.J. Lavernia, Acta Mater. 51 (2003) 2.777-2.791.

[20] J. Lee, F. Zhou, K.H. Chung, N.J. Kim y E.J. Lavernia, Met. Mater. Trans A 32 (2001) 3.109. 3.115 .

[21] K. Chung y E.J. Lavernia, Met. Mater. Trans. A 33 (2002) 3.795-3.801.

[22] K. Chung, J. Lee, R. Rodríguez, y E.J. Lavernia, Met. Mater. Trans A 33 (2002) 125-134. 